\title{
Social media data mining and knowledge discovery under wireless network
}

\author{
Xiaoxian Yang ${ }^{1} \cdot$ Li Kuang $^{2}$
}

Accepted: 23 February 2021 / Published online: 11 March 2021

(C) The Author(s), under exclusive licence to Springer Science+Business Media, LLC, part of Springer Nature 2021

The prosperity of social media brings revolutionary changes to our daily lives and generates a huge volume of data as well. Mining and analyzing social media big data gives us the opportunity to understand how people communicate, interact, and collaborate online. Moreover, it is also useful for designers to improve social media systems for serving people needs better. As a result, social media big data mining and analytics has been one of the hottest research areas and attracting more and more endeavor from the research community. We receive many submissions and finally select six papers for this special issue. A summary of these papers is outlined below.

In the paper entitled "Road Network Layout Based Multi-hop Broadcast Protocols for Urban Vehicular Adhoc Networks" by Lazhar Khamer et al., two new routing broadcast protocols are proposed to support different services such as road safety, traffic efficiency, entertainment, and advertisement in the decentralized wireless Vehicular Ad hoc Networks (VANETs). The proposed protocols are the Enhanced Counter-based broadcast protocol in Urban VANET (ECUV) and the Enhanced distance-based broadcast protocol in Urban VANET (EDUV). The network simulation results show clearly that ECUV and EDUV outperform other methods in terms of coverage capacity and efficiency.

In the paper entitled "Transformation-based Processing of Typed Resources for Multimedia Sources in the IoT Environment" by Honghao Gao et al., the authors cope with the challenge of how to manage a variety of web

Xiaoxian Yang

xxyang@sspu.edu.cn

Li Kuang

kuangli@csu.edu.cn

1 School of Computer and Information Engineering, Shanghai Polytechnic University, Shanghai, China

2 School of Computer Science and Engineering, Central South University, Changsha, China services, and they present an extension of DIKW (Data, Information, Knowledge, and Wisdom) architecture as a resource expression model to construct a systematic approach to modeling both entity and relationship elements. The proposed method improves the optimization of services and scheduling resources of multimedia systems.

In the paper entitled "Improving the Classification of Call Center Service Dialogue with Key Utterences" by Yuqi Liu et al., the authors propose a dialogue classification algorithm that strengthens the influence of the business-related utterances in the dialogue and use them as the key utterances to improve the classification. Firstly, key utterance labels that indicate which utterances in the dialogue are key utterances are proposed. Then, the authors propose the dialogue classification model based on the key utterance labels and logistic regression, namely KU-LR. The experimental results on the real-world dataset show that the KU-LR method outperforms other baselines when the training dataset is small.

In the paper entitled "Potential Trend Discovery for Highway Drivers on Spatio-temporal Data" by Weilong Ding et al., a travel-characteristic-based method is proposed to discover the potential trend of payment identity for highway drivers. Considering time, space, subjective preference, and objective property, travel characteristics are modeled on monthly data from highway toll stations, through which predictive errors can be reduced by gradient boosting classification. With real-world data of one Chinese provincial highway network, extensive experiments and case studies show that the proposed method has second-level executive latency with more than $85 \%$ F1-score for trend discovery.

In the paper entitled "A Mobility Aware Duty Cycling and Preambling Solution for Wireless Sensor Network with Mobile Sink Node" by Craig Thomson et al., the Mobility Aware Duty Cycling and Dynamic Preambling Algorithm (MADCaDPAL) is proposed. This algorithm utilizes an existing solution where a communication threshold is predefined between a mobile sink node using 
predictable mobility and static nodes on its path. MADCaDPAL bases decisions relating to node sleep function, moving to clear channel assessment, and the subsequent sending of preambles on the relation between the threshold built by the static node and the position of the mobile sink node. MADCaDPAL achieves a reduction in average energy consumption of up to $80 \%$ when used in conjunction with a lightweight carrier-sense multiple access-based MAC implementation.

In the paper entitled "Preference Discovery from Wireless Social Media Data in APIs Recommendation" by Yueshen $\mathrm{Xu}$ et al., the authors discover that there is a relationship between the user and the API, and they use such relationships and collaborative learning techniques to finish APIs recommendation. The authors propose a holistic framework that contains three models, including two modes on the user's side and APIs side, and an ensemble model fully leveraging the preference mined from both user's side and APIs side. The experiment results on a real-world dataset show that the proposed models perform better than all compared methods.

The Guest Editors would like to express their deep gratitude to all the authors who have submitted their valuable contributions, and to the numerous and highly qualified anonymous reviewers. We also would like to thank the support from the National Natural Science Foundation of China (No. 61902236). We think that the selected contributions, which represent the current state of the art in the field, will be of great interest to the community.

Publisher's Note Springer Nature remains neutral with regard to jurisdictional claims in published maps and institutional affiliations.

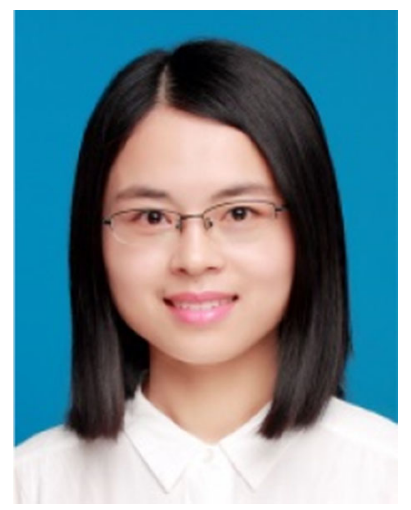

Xiaoxian Yang received a Ph.D. in Management Science and Engineering from Shanghai University, China, in 2017. She is currently an assistant professor at Shanghai Polytechnic University, China. Dr. Yang's research interests include business process management, formal verification, wireless networks, and mobile health. She has published more than 20 papers in academic journals such as IEEE TITS, IEEE TCSS, ACM TOMM, ACM TOIT, FGCS, MONET, IJSEKE, FGCS, COMNET. She has obtained 2 patents and 3 registered software copyrights in China involving wireless networks, workflow management, and formal verification. Dr. Yang has participated in organizing international conferences and workshops, such as CollaborateCom 2018-2020, ChinaCom 2019-2020, and Mobicase 2019-2020. She also worked as a guest editor for MONET, WINE and JIT and served as a reviewer for IEEE TII, IEEE T-ITS, IEEE T-ASE, IEEE JBHI, FGCS, PPNA, Wireless Networks, Computer Networks, etc.

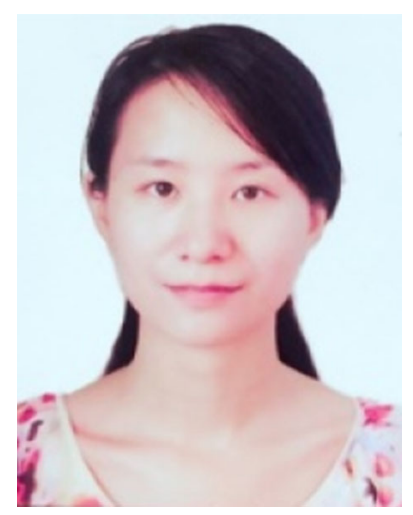

Li Kuang received a Ph.D. in Computer Science from Zhejiang University, China, in 2009. She is currently a professor at the School of Computer Science and Engineering, Central South University. Her research interests include service computing, mobile computing and privacy preservation. 